\title{
DISCUSSION
}

\section{Analysis of coupled heat, moisture and air transfer in a deformable unsaturated soil}

\author{
H. R. THOMAS and Y. HE (1995). Géotechnique 45, No. 4, 677-689.
}

\section{Q. Yang and E. C. Leong, Nanyang Techno- logical University, Singapore}

The authors have presented an interesting paper on the analysis of coupled heat, moisture and air transfer in deformable unsaturated soils. Their work has extended previous analyses of heat and moisture transfer in unsaturated soils to take account of deformation and stress-strain behaviour. The authors have used elasticity theory coupled with the so-called state surface approach to relate volumetric strain not only to stress, but also to suction and temperature changes. As shown in the paper, strong interactions occur between heat and mois- ture flows and the deformation behaviour of the unsaturated soil. Owing to the complexity and the highly non-linear nature of the problem, however, discussion is needed on the energy conservation of heat and on thermally induced moisture migration.

In performing analyses of coupled heat, moisture and air transfer in deformable unsaturated soils, it is important to achieve mass conservation of moisture and energy conservation of heat, both in the theoretical formulation and in the numerical implementation. In the energy conservation equation (4) in the paper, the heat content of the soil $\Phi$ consists of two terms: one represents the storage of latent heat due to the accumulation of vapour and the other represents the local capacity for heat comprising the capacity of the soil solids, the pore water, the pore water vapour and the pore dry air. Within the local capacity term, in the component due to the soil solids $(1-n) \rho_{\mathrm{s}} C_{\mathrm{ps}}, n$ is the porosity that varies with volumetric deformation of the soil skeleton, and $\rho_{\mathrm{s}}$ and $C_{\mathrm{ps}}$ are the density and the specific heat capacity respectively of the soil solids. The theoretical formulation of the energy conservation equation in the paper is correct. However, we would like to point out a discrepancy in the numerical implementation of the energy conservation equation by using the finite element method. Using a simple numerical example of an ideal unit of soil loaded under undrained and adiabatic conditions (Fig. 1), the computed temperature decreases with decrease in porosity of the soil in order to satisfy the conservation of energy equation. This is not correct as there is no heat change in the soil, and therefore the temperature in the soil must also remain constant. In order to clarify this, we have to look at the finite element formulation for the calculation of the mass of the soil solids. The mass of the soil solids in the given region $\Omega$ (Fig. 1) can be simply calculated as

$$
M=\iint_{\Omega}(1-n) \rho_{\mathrm{s}} \mathrm{d} S
$$

where $n=e /(1+e)$. In Fig. $1, \Omega_{0}$ represents the initial geometrical region with an area of $\left(1+e_{0}\right)$ and $\Omega$ represents the final geometrical region with an area of $(1+e)$, where $e_{0}$ and $e$ are the initial void ratio and the final void ratio respectively. The thickness of the ideal unit of the soil is assumed to be 1. Application of equation (1) to the case of Fig. 1 shows that the initial total mass of the soil solids $M_{0}$ is the same as the final mass, that is, $\rho_{\mathrm{s}}$. But in the conventional finite element calculation, the element meshes are fixed, therefore the geometrical region of integration is fixed accordingly. The final total mass of the soil solids calculated by the finite element method is $\left(1+e_{0}-e\right) \rho_{\mathrm{s}}$, which is larger than the initial mass if $e$ is less than $e_{0}$. In our experience, the numerical discrepancy in the temperature due to the numerical discrepancy in the mass of the soil solids can be as high as $10^{\circ} \mathrm{C}$ in some cases. In order to correct for this situation, it

$q$

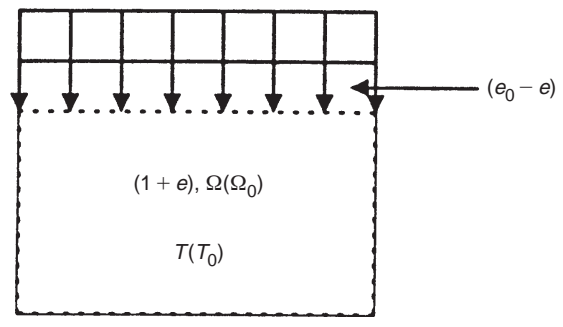

Fig. 1. Ideal unit of soil under undrained conditions and non-exchange of heat 
is suggested that one replaces the local capacity of the soil solids $(1-n) \rho_{\mathrm{s}} C_{\mathrm{ps}}$ with $\left(1-n_{0}\right) \rho_{\mathrm{s}} C_{\mathrm{ps}}$ in the numerical modelling.

With regard to the moisture transfer in the heating experiment test, we agree with the authors' numerical results which revealed that thermally induced moisture migration causes both liquid and vapour to be driven away from the heater, and liquid transfer provides a greater component of moisture flow from the heater than vapour transfer. However, on p. 679 of the paper, the velocity equation (8) of liquid flow does not contain a term consisting of the temperature gradient. If it is true that a temperature gradient will give rise to a pore vapour pressure gradient and a pore air pressure gradient that cause liquid water to be driven away from the heater, then the temperature gradient should be included in the velocity equation (8). Perhaps the authors can clarify this aspect.

\section{Authors' reply}

The authors thank the contributors for their interest and comments on the paper. The problem analysed is indeed complex in nature, but limitations of space associated with the publication of one paper unfortunately prevent detailed consideration of all aspects of the work. The authors therefore welcome discussion of the points raised.

The authors' paper presents an analysis of coupled heat, moisture and air transfer, which is applicable to a deformable, unsaturated soil. The heat transfer side of the problem accommodates thermal conduction, sensible heat transfer associated with liquid, vapour and air flow, and latent heat flow with vapour. The heat content of the soil consequently consists of the cumulative effect of the storage of latent heat due to the accumulation of vapour and the local capacity for heat comprising the capacity of soil solids, soil water, the soil water vapour and soil dry air.

The contributors focus attention on only one term of the heat content expression and consider the effect of deformation on that term alone. Since the specific heat capacity and the density of the solid particles remain constant, the problem addressed by the contributors essentially degenerates to the correct calculation of the mass of the solid particles using the finite element method.

We agree that careful consideration must be given to the numerical implementation of the energy conservation equation, and confirm that the phenomenon described by the contributors was also observed by us. However, we wish to emphasize that the problem posed by the contributors is an extreme case of a completely dry soil. In the examples considered by us, namely unsaturated soil containing liquid, vapour and dry air, the heat content is influenced not only by changes in solid particle content, but also by variations in the other phases. The combined effect of all phases of the soil is thus accommodated in a realistic fashion. Equation (23) in the paper presents the governing differential equation for energy conservation. From this equation the relationship between $\partial \Phi / \partial t$ and $\partial n / \partial t$ can be established, from which it can be seen that the effect of changes in the soil solids phase act essentially in the opposite direction to the effect of changes in the other phases. It can thus be concluded that the overall effect of the phenomenon highlighted by the contributors is reduced when the other phases of an unsaturated soil are included.

The contributors' suggested solution appears simply to set the $\partial n / \partial t$ terms in equation (23) to zero, effectively removing this aspect of the coupling of the heat transfer and deformation equations.

For reasons of limitation of space, as outlined above, the paper does not consider in detail the authors' numerical formulation of the problem. Further details are, however, available (Thomas \& Ye, 1997).

Regarding the components of moisture transfer included in the formulation, we reaffirm that thermally induced liquid and vapour transfer are both included in the formulation. Equation (8), presented on p. 679, provides a basic definition of moisture velocity, which inherently contains the effect of a temperature gradient, should one exist. The method by which equation (8) is developed to accommodate components of flow due to a moisture content and temperature gradient (Ewen \& Thomas, 1989) or a potential and temperature gradient (Thomas \& King, 1991) has been presented in detail previously by the senior author and co-workers. Again, due to limitations of space, it was not considered appropriate to repeat all the details in this paper. The approach adopted is based on the fact that the potential for flow varies with both moisture content and temperature, with the latter included by way of surface energy effects.

\section{REFERENCES}

Ewen, J. \& Thomas, H. R. (1989). Heating unsaturated medium sand. Géotechnique 39, No. 3, 455-470.

Thomas, H. R. \& He, Y. (1997). A coupled heatmoisture transfer theory for deformable unsaturated soil and its algorithmic implementation. Accepted for publication in Int. J. Numer. Methods in Engng.

Thomas, H. R. \& King, S. D. (1991). Coupled temperature/capillary potential variations in unsaturated soil. J. Engng Mech., Am. Soc. Civ. Engrs 117, No. 11, 2475-2491. 